\title{
Similarity Solution for Fractional Diffusion Equation
}

\author{
Jun-Sheng Duan, ${ }^{1}$ Ai-Ping Guo, ${ }^{2}$ and Wen-Zai Yun ${ }^{2}$ \\ ${ }^{1}$ School of Sciences, Shanghai Institute of Technology, Shanghai 201418, China \\ ${ }^{2}$ School of Mathematics, Baotou Teachers College, Baotou, Inner Mongolia 014030, China
}

Correspondence should be addressed to Jun-Sheng Duan; duanjs@sit.edu.cn

Received 4 January 2014; Accepted 11 February 2014; Published 12 March 2014

Academic Editor: Ming Li

Copyright (C) 2014 Jun-Sheng Duan et al. This is an open access article distributed under the Creative Commons Attribution License, which permits unrestricted use, distribution, and reproduction in any medium, provided the original work is properly cited.

Fractional diffusion equation in fractal media is an integropartial differential equation parametrized by fractal Hausdorff dimension and anomalous diffusion exponent. In this paper, the similarity solution of the fractional diffusion equation was considered. Through the invariants of the group of scaling transformations we derived the integro-ordinary differential equation for the similarity variable. Then by virtue of Mellin transform, the probability density function $p(r, t)$, which is just the fundamental solution of the fractional diffusion equation, was expressed in terms of Fox functions.

\section{Introduction}

Standard diffusion in $d$-dimensional space, where $d$ is a positive integer, is a process described by Gaussian distribution. A main feature of the process is the linear relation between the mean square displacement and time; namely, $\left\langle r^{2}(t)\right\rangle \propto$ $t$. Some anomalous diffusion phenomena that take place in impure media, biological tissues, and porous media can be simulated by the diffusion model in fractals [1-6]. In recent years, the fractal theory has been developed rapidly, and it was found to be closely related to the anomalous diffusion phenomena [3-12].

In fractal media, the geometric obstacles existing on all length scales slow down the particle motion in a random walk. The mean square displacement behaves as [2]

$$
R^{2} \equiv\left\langle r^{2}(t)\right\rangle \propto t^{2 / d_{w}},
$$

where $d_{w}(>2)$ is the anomalous diffusion exponent. The numerical simulation found that on a large class of fractal structures the general form of the probability density function $p(r, t)$ that the walker is at distance $r$ at time $t$ from its starting point at time $t=0$ obeys asymptotically a nonGaussian shape of the form $[2,3]$

$$
p(r, t) \sim t^{-d_{f} / d_{w}} \exp \left[- \text { const. } \times\left(\frac{r}{R}\right)^{u}\right], \quad \frac{r}{R} \gg 1,
$$

where $u=d_{w} /\left(d_{w}-1\right)$ and $d_{f}$ is the fractal Hausdorff dimension.

In order to simulate the diffusion phenomena in fractal media, some scholars have introduced fractional diffusion equations $[4,5,11-13]$. In this paper, we consider the fractional diffusion equation $[5,13]$ :

$$
\frac{\partial^{\gamma} p(r, t)}{\partial t^{\gamma}}=\frac{1}{r^{d_{s}-1}} \frac{\partial}{\partial r}\left(r^{d_{s}-1} \frac{\partial p}{\partial r}\right), \quad r>0, t>0,
$$

where $\gamma=2 / d_{w}, d_{s}=2 d_{f} / d_{w}$ is the spectral dimension of the fractal, and the fractional time derivative on the left hand side of (3) is defined as the convolution integral [14-20]:

$$
\frac{\partial^{\gamma} p(r, t)}{\partial t^{\gamma}}=\frac{\partial}{\partial t} \int_{0}^{t} \frac{(t-\tau)^{-\gamma}}{\Gamma(1-\gamma)} p(r, \tau) d \tau, \quad 0<\gamma<1,
$$

where $\Gamma(\cdot)$ is Euler's gamma function. In the limit case, $d_{w} \rightarrow$ 2 and $d_{f} \rightarrow d$, (3) reduces to the standard $d$-dimensional diffusion equation.

The fractional calculus has been applied to many fields in science and engineering, such as viscoelasticity, anomalous diffusion, biology, chemistry, and control theory $[5,11-13$, $15,19-22]$. Researches on the fractional differential equations attract much attention [15, 23-28]. For linear fractional differential equations, the integral transforms, including the 
Laplace, Fourier, and Mellin transforms, are usually used to obtain analytic solutions.

In this paper using the similarity method [29] we solve (3) with the following initial and boundary conditions and the conservation condition:

$$
\begin{gathered}
p(r, 0)=0, \quad r>0, \\
p(\infty, t)=0, \quad t>0, \\
\omega\left(d_{f}\right) \int_{0}^{\infty} p(r, t) r^{d_{f}-1} d r=1,
\end{gathered}
$$

where $\omega\left(d_{f}\right)$ is a constant, which is defined as

$$
\omega\left(d_{f}\right)=\frac{2 \pi^{d_{f} / 2}}{\Gamma\left(d_{f} / 2\right)} .
$$

We note that the probability density function $p(r, t)$ is just the fundamental solution of the fractional diffusion equation. The similarity method was used by Gorenflo et al. [30], Wyss [31], and Buckwar and Luchko [32] for solving problems of time fractional partial differential equations in one-dimensional case.

\section{Derivation of Similarity Solution}

First we determine a symmetric group of scaling transformations

$$
T_{\alpha}: r=\alpha \bar{r}, \quad t=\alpha^{h} \bar{t}, \quad p=\alpha^{l} \bar{p},
$$

where $\alpha>0$ is a parameter and $h, l$ are constants to be determined. Applying the group of scaling transformations (7), the fractional derivative is converted as follows:

$$
\begin{aligned}
\frac{\partial^{\gamma} p(r, t)}{\partial t^{\gamma}} & =\alpha^{l} \frac{\partial}{\partial t} \int_{0}^{t} \frac{(t-\tau)^{-\gamma}}{\Gamma(1-\gamma)} \bar{p}\left(\bar{r}, \alpha^{-h} \tau\right) d \tau \\
& =\alpha^{l-h \gamma} \frac{\partial}{\partial \bar{t}} \int_{0}^{\bar{t}} \frac{\left.\bar{t}-\tau^{\prime}\right)^{-\gamma}}{\Gamma(1-\gamma)} \bar{p}\left(\bar{r}, \tau^{\prime}\right) d \tau^{\prime} \\
& =\alpha^{l-h \gamma} \frac{\partial^{\gamma} \bar{p}(\bar{r}, \bar{t})}{\partial \bar{t}^{\gamma}}
\end{aligned}
$$

where $\tau^{\prime}=\alpha^{-h} \tau$. Hence the problem (3)-(5) is invariant under the group (7) if and only if

$$
h=\frac{2}{\gamma}, \quad l=-d_{f} .
$$

So the symmetric group of scaling transformations is determined:

$$
T_{\alpha}: r=\alpha \bar{r}, \quad t=\alpha^{2 / \gamma} \bar{t}, \quad p=\alpha^{-d_{f}} \bar{p} .
$$

Eliminating the parameter $\alpha$ leads to two invariants:

$$
r t^{-\gamma / 2}=\bar{r} \bar{t}^{-\gamma / 2}, \quad r^{d_{f}} p=\bar{r}^{d_{f}} \bar{p} .
$$

We denote the two invariants of the group of the scaling transformation $T_{\alpha}$ as

$$
\eta=r t^{-\gamma / 2}, \quad F=r^{d_{f}} p .
$$

Next we use the transformation

$$
p(r, t)=r^{-d_{f}} F(\eta), \quad \eta=r t^{-\gamma / 2}
$$

to determine the equations for the similarity solution of the problem (3)-(5). Calculating derivative we have

$$
\begin{gathered}
\frac{\partial p}{\partial r}=-d_{f} r^{-d_{f}-1} F(\eta)+r^{-d_{f}} t^{-\gamma / 2} F^{\prime}(\eta) \\
\frac{1}{r^{d_{s}-1}} \frac{\partial}{\partial r}\left(r^{d_{s}-1} \frac{\partial p}{\partial r}\right)=r^{-d_{f}} t^{-\gamma} F^{\prime \prime}(\eta)+\Delta_{1} r^{-d_{f}-1} t^{-\gamma / 2} F^{\prime}(\eta) \\
+\Delta_{2} r^{-d_{f}-2} F(\eta),
\end{gathered}
$$

where

$$
\Delta_{1}=d_{s}-1-2 d_{f}, \quad \Delta_{2}=d_{f}\left(d_{f}-d_{s}+2\right) .
$$

For the left hand side of (3), we introduce the new integral variable

$$
\xi=r \tau^{-\gamma / 2},
$$

we obtain $p(r, \tau)=r^{-d_{f}} F(\xi)$, and

$$
\begin{aligned}
& \frac{\partial^{\gamma} p(r, t)}{\partial t^{\gamma}}=\frac{\partial}{\partial t} \int_{0}^{t} \frac{(t-\tau)^{-\gamma}}{\Gamma(1-\gamma)} p(r, \tau) d \tau \\
&=-r^{2 / \gamma^{-} d_{f}-1} t^{-\gamma / 2-1} \frac{d}{d \eta} \int_{\eta}^{+\infty} \frac{\left[(\eta / \xi)^{-2 / \gamma}-1\right]^{-\gamma}}{\Gamma(1-\gamma)} \\
& \times F(\xi) \xi^{1-2 / \gamma} d \xi .
\end{aligned}
$$

Letting

$$
g(w)= \begin{cases}\frac{\left(w^{-2 / \gamma}-1\right)^{-\gamma}}{\Gamma(1-\gamma)}, & 0<w<1, \\ 0, & w>1,\end{cases}
$$

we rewrite (18) as

$$
\frac{\partial^{\gamma} p(r, t)}{\partial t^{\gamma}}=-r^{2 / \gamma^{-} d_{f}-1} t^{-2 / \gamma-1} \frac{d}{d \eta} \int_{0}^{+\infty} g\left(\frac{\eta}{\xi}\right) F(\xi) \xi^{1-2 / \gamma} d \xi .
$$

From (15) and (20), we obtain the integro-ordinary differential equation for the similarity variables:

$$
\begin{aligned}
& -\frac{d}{d \eta} \int_{0}^{+\infty} g\left(\frac{\eta}{\xi}\right) F(\xi) \xi^{-2 / \gamma+1} d \xi \\
& \quad=\eta^{-2 / \gamma+1} F^{\prime \prime}(\eta)+\Delta_{1} \eta^{-2 / \gamma} F^{\prime}(\eta)+\Delta_{2} \eta^{-2 / \gamma-1} F(\eta) .
\end{aligned}
$$

The conditions (5) are converted to

$$
F(+\infty)=0, \quad \omega\left(d_{f}\right) \int_{0}^{+\infty} F(\eta) \eta^{-1} d \eta=1 .
$$

Considering the integration in (21), we use Mellin transforms for the new problem (21) and (22). The Mellin transform of function $f(x)$ is defined as [33]

$$
\widehat{f}(s)=\mathscr{M}[f(x), s]=\int_{0}^{+\infty} f(x) x^{s-1} d x .
$$

Applying Mellin transform with respect to $\eta$ to both sides of (21), we get

$$
(s-1) \hat{g}(s-1) \widehat{F}\left(s-\frac{2}{\gamma}+1\right)
$$




$$
\begin{aligned}
& =\left(\left(s-\frac{2}{\gamma}\right)\left(s-\frac{2}{\gamma}-1\right)-\Delta_{1}\left(s-\frac{2}{\gamma}-1\right)+\Delta_{2}\right) \\
& \quad \times \widehat{F}\left(s-\frac{2}{\gamma}-1\right) .
\end{aligned}
$$

Calculating integrations we obtain Mellin transform of the function $g(w)$ :

$$
\widehat{g}(s)=\frac{\Gamma(\gamma+\gamma s / 2)}{s \Gamma(\gamma s / 2)} .
$$

Inserting (25) into (24) and then replacing $s$ by $s+2 / \gamma+1$ we obtain the difference equation for the function $\widehat{F}(s)$ :

$$
\frac{\Gamma(\gamma(s / 2+1 / \gamma+1))}{\Gamma(\gamma(s / 2+1 / \gamma))} \widehat{F}(s+2)=\left(s^{2}+s-\Delta_{1} s+\Delta_{2}\right) \widehat{F}(s) .
$$

In order to solve the difference equation, we introduce $s=$ $2 q$ and $\widehat{F}(2 q)=T(q)$, and rewrite (26) into

$$
\frac{T(q+1)}{T(q)}=4\left(q+\frac{d_{f}}{2}\right)\left(q+1+\frac{d_{f}-d_{s}}{2}\right) \frac{\Gamma(\gamma q+1)}{\Gamma(\gamma q+\gamma+1)} .
$$

A particular solution of (27) is

$$
T(q)=C \frac{4^{q} \Gamma\left(q+d_{f} / 2\right) \Gamma\left(q+1+\left(d_{f}-d_{s}\right) / 2\right)}{\Gamma(\gamma q+1)},
$$

where $C$ is an arbitrary constant. For the solution of (27), we can multiply $T(q)$ by any function $Y(q)$ which satisfies $Y(q+$ 1) $/ Y(q)=1$.

We notice that $\widehat{F}(s)$ is a Mellin transform defined only in some strip $0 \leq \sigma_{1}<\operatorname{Re}(s)<\sigma_{2}$ from the conditions (22). So (26) is valid only in the overlap of the two strips $\sigma_{1}<\operatorname{Re}(s)<$ $\sigma_{2}$ and $\sigma_{1}<\operatorname{Re}(s+2)<\sigma_{2}$, and there is no such overlap unless $\sigma_{1}+2<\sigma_{2}$. Thus $Y(q)$ cannot have poles; otherwise, it would have a row of poles separated exactly by one unit. In addition, $Y(q)$ cannot grow faster than $|q|$ as $\operatorname{Im}(q) \rightarrow \infty$ in the inversion strip; otherwise the inversion integral would diverge. Thus $Y(q)$ is a bounded entire function and equals a constant by Liouville's theorem.

Therefore, $T(q)$ has only the form of (28) and we have

$$
\begin{aligned}
\widehat{F}(s) & =T\left(\frac{s}{2}\right) \\
& =C \frac{2^{s} \Gamma\left(d_{f} / 2+s / 2\right) \Gamma\left(1+\left(d_{f}-d_{s}\right) / 2+s / 2\right)}{\Gamma(1+\gamma s / 2)} .
\end{aligned}
$$

It follows from (22) that $\widehat{F}(0)=1 / \omega\left(d_{f}\right)$. Thus we have

$$
C=\frac{1}{\omega\left(d_{f}\right) \Gamma\left(d_{f} / 2\right) \Gamma\left(1+\left(d_{f}-d_{s}\right) / 2\right)} .
$$

The inverse Mellin transform of (29) is

$F(\eta)$

$$
\begin{aligned}
=\frac{C}{2 \pi i} \int_{c-i \infty}^{c+i \infty} \frac{2^{s} \Gamma\left(d_{f} / 2+s / 2\right) \Gamma\left(1+\left(d_{f}-d_{s}\right) / 2+s / 2\right)}{\Gamma(1+\gamma s / 2)} \\
\times \eta^{-s} d s .
\end{aligned}
$$

Replacing $s$ by $-s$ and using the definition of Fox functions we obtain $[34,35]$

$$
F(\eta)=C H_{1,2}^{2,0}\left(\left.\frac{\eta}{2}\right|_{\left(d_{f} / 2,1 / 2\right),\left(1+\left(d_{f}-d_{s}\right) / 2,1 / 2\right)} ^{(1, \gamma / 2)}\right) .
$$

Inserting the expressions into (13) and using properties of Fox functions, we obtain the probability density function in terms of the Fox function:

$$
\begin{aligned}
p(r, t)= & \frac{d_{w} 2^{-d_{f}} t^{-d_{f} / d_{w}}}{\omega\left(d_{f}\right) \Gamma\left(d_{f} / 2\right) \Gamma\left(1+d_{f} / 2-d_{f} / d_{w}\right)} \\
& \times H_{1,2}^{2,0}\left(\left.\frac{r^{d_{w}}}{2^{d_{w} t}}\right|_{\left(0, d_{w} / 2\right),\left(1-d_{f} / d_{w}, d_{w} / 2\right)} ^{\left(1-d_{f} / d_{w}, 1\right)}\right) .
\end{aligned}
$$

For a large class of fractal structures, the spectral dimension [2] satisfies $d_{s}<2$; that is, $d_{f}<d_{w}$. So the Fox function in (33) can be expanded into a series by using residue theorem on the simple poles:

$$
P_{a}=\left\{\frac{2 k}{d_{w}} \mid k=0,1, \ldots\right\} \cup\left\{\frac{2}{d_{w}}\left(1-\frac{d_{f}}{d_{w}}+k\right) \mid k=0,1, \ldots\right\} .
$$

The series representation for the probability density is calculated to be

$$
\begin{aligned}
& p(r, t) \\
& =\frac{2^{1-d_{f}} t^{-d_{f} / d_{w}}}{\omega\left(d_{f}\right) \Gamma\left(d_{f} / 2\right) \Gamma\left(1+d_{f} / 2-d_{f} / d_{w}\right)} \\
& \times \sum_{k=0}^{\infty} \frac{(-1)^{k}}{k !} \times\left[\frac{\Gamma\left(1-d_{f} / d_{w}-k\right)}{\Gamma\left(1-d_{f} / d_{w}-2 k / d_{w}\right)}\left(\frac{r^{d_{w}}}{2^{d_{w}}}\right)^{2 k / d_{w}}\right. \\
& + \\
& \quad \frac{\Gamma\left(d_{f} / d_{w}-1-k\right)}{\Gamma\left(1-d_{f} / d_{w}-2 / d_{w}\left(1-d_{f} / d_{w}+k\right)\right)} \\
& \left.\quad \times\left(\frac{r^{d_{w}}}{2^{d_{w} t}}\right)^{2 / d_{w}\left(1-d_{f} / d_{w}+k\right)}\right] .
\end{aligned}
$$




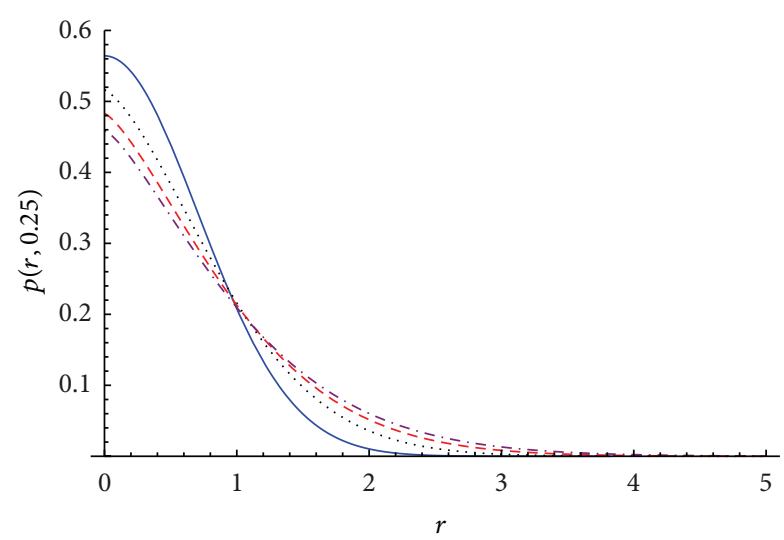

Figure 1: Curves of $p(r, 0.25)$ versus $r$ for $d_{f}=1$ and for $d_{w}=2$ (solid line), $d_{w}=2.5$ (dot line), $d_{w}=3$ (dash line), and $d_{w}=3.5$ (dot-dash line).

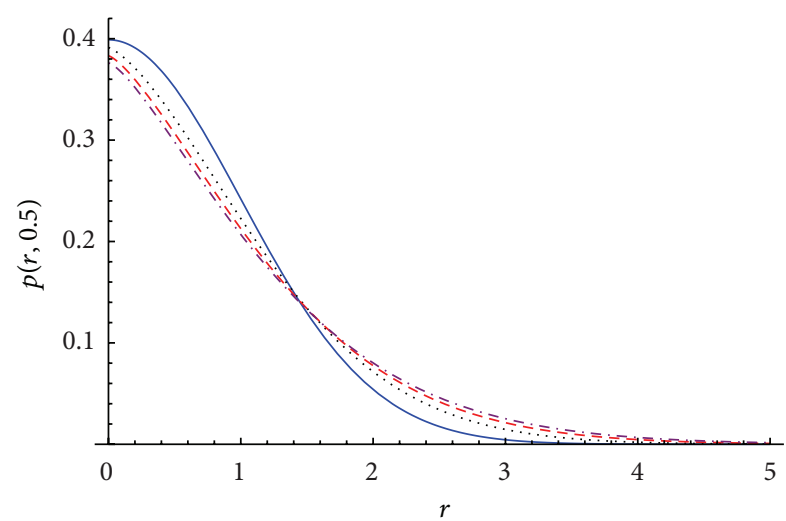

Figure 2: Curves of $p(r, 0.5)$ versus $r$ for $d_{f}=1$ and for $d_{w}=2$ (solid line), $d_{w}=2.5$ (dot line), $d_{w}=3$ (dash line), and $d_{w}=3.5$ (dot-dash line).

\section{Discussions and Conclusions}

In the limit case, $d_{w} \rightarrow 2$ and $d_{f} \rightarrow d$, (3) reduces to the $d$ dimensional standard diffusion equation, and the probability density (35) is simplified to the Gaussian distribution:

$$
p(r, t)=\frac{1}{(4 \pi t)^{d / 2}} \exp \left(-\frac{r^{2}}{4 t}\right) .
$$

In Figures 1 and 2 , we plot the curves of $p(r, 0.25)$ versus $r$ and $p(r, 0.5)$ versus $r$, respectively, for $d_{f}=1$ and different values of $d_{w}$. In Figures 3 and 4 , we plot the curves of $p(r, 0.25)$ versus $r$ and $p(r, 0.5)$ versus $r$, respectively, for $d_{f}=$ 1.5 and different values of $d_{w}$. The figures display that, as the anomalous diffusion exponent $d_{w}$ increases, the peak value of the probability density function $p(r, t)$ at $r=0$ decreases. In addition, as the fractal Hausdorff dimension $d_{f}$ increases from 1 to 1.5 , the peak value of $p(r, t)$ at $r=0$ decreases.

Compared with the similarity method for classic partial differential equations, the similarity method for fractional diffusion equation involves the similarity integral variable $\xi=r \tau^{-\gamma / 2}$, and the reduction equation is an integro-ordinary differential equation for the similarity solution. The obtained

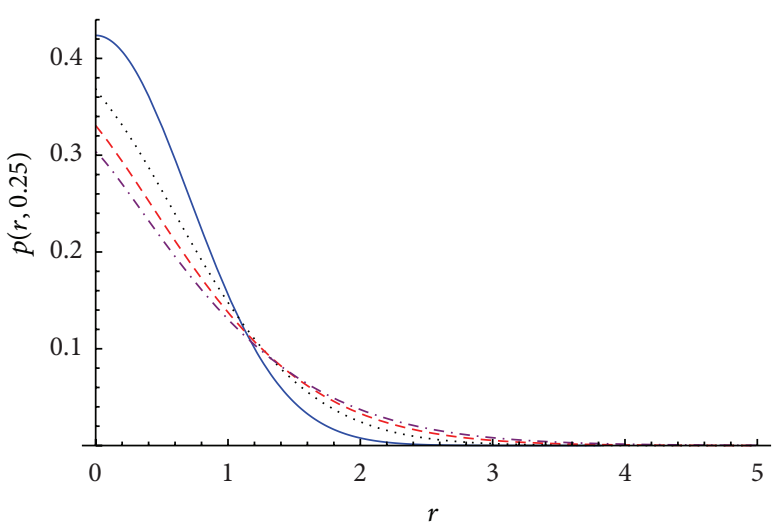

Figure 3: Curves of $p(r, 0.25)$ versus $r$ for $d_{f}=1.5$ and for $d_{w}=2$ (solid line), $d_{w}=2.5$ (dot line), $d_{w}=3$ (dash line), and $d_{w}=3.5$ (dot-dash line).

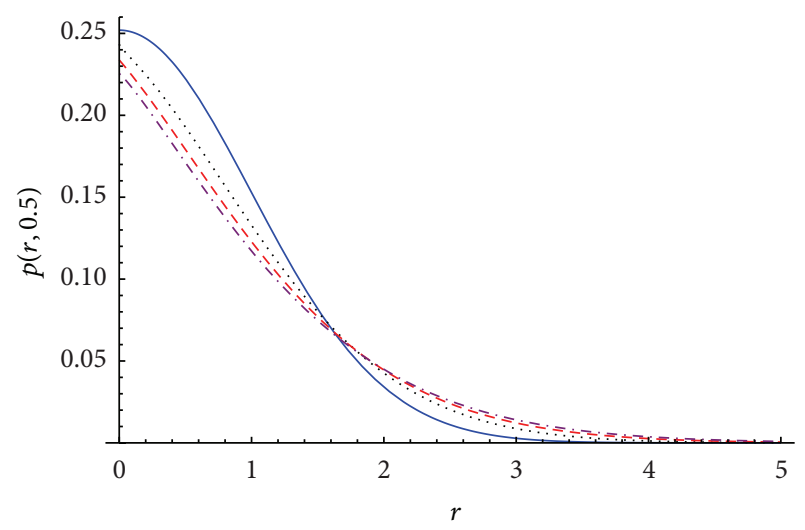

FIGURE 4: Curves of $p(r, 0.5)$ versus $r$ for $d_{f}=1.5$ and for $d_{w}=2$ (solid line), $d_{w}=2.5$ (dot line), $d_{w}=3$ (dash line), and $d_{w}=3.5$ (dot-dash line).

probability density $p(r, t)$ is just the fundamental solution of the fractional diffusion equation.

\section{Conflict of Interests}

The authors declare that there is no conflict of interests regarding the publication of this paper.

\section{Acknowledgments}

This work was supported by the National Natural Science Foundation of China (11201308) and the Innovation Program of Shanghai Municipal Education Commission (14ZZ161).

\section{References}

[1] B. B. Mandelbrot, The Fractal Geometry of Nature, W. H. Freeman, New York, NY, USA, 1982.

[2] S. Havlin and D. Ben-Avraham, "Diffusion in disordered media," Advances in Physics, vol. 51, no. 1, pp. 187-292, 2002.

[3] D. Liu, H. Li, F. Chang, and L. Lin, "Anomalous diffusion on the percolating networks," Fractals, vol. 6, no. 2, pp. 139-144, 1998. 
[4] F.-Y. Ren, J.-R. Liang, and X.-T. Wang, "The determination of the diffusion kernel on fractals and fractional diffusion equation for transport phenomena in random media," Physics Letters A, vol. 252, no. 3-4, pp. 141-150, 1999.

[5] Q. Zeng and H. Li, "Diffusion equation for disordered fractal media," Fractals, vol. 8, no. 1, pp. 117-121, 2000.

[6] C. Cattani, "Fractals and hidden symmetries in DNA," Mathematical Problems in Engineering, vol. 2010, Article ID 507056, 31 pages, 2010.

[7] M. Li and W. Zhao, "On bandlimitedness and lag-limitedness of fractional Gaussian noise," Physica A, vol. 392, no. 9, pp. 19551961, 2013.

[8] M. Li, "A class of negatively fractal dimensional Gaussian random functions," Mathematical Problems in Engineering, vol. 2011, Article ID 291028, 18 pages, 2011.

[9] M. Li, C. Cattani, and S.-Y. Chen, "Viewing sea level by a onedimensional random function with long memory," Mathematical Problems in Engineering, vol. 2011, Article ID 654284, 13 pages, 2011.

[10] C. Cattani and G. Pierro, "On the fractal geometry of DNA by the binary image analysis," Bulletin of Mathematical Biology, vol. 75, no. 9, pp. 1544-1570, 2013.

[11] R. Metzler and J. Klafter, “The random walk's guide to anomalous diffusion: a fractional dynamics approach," Physics Reports, vol. 339, no. 1, pp. 1-77, 2000.

[12] M. Giona and H. E. Roman, "Fractional diffusion equation for transport phenomena in random media," Physica A, vol. 185, no. 1-4, pp. 87-97, 1992.

[13] R. Metzler, W. G. Glöckle, and T. F. Nonnenmacher, "Fractional model equation for anomalous diffusion," Physica A, vol. 211, no. 1, pp. 13-24, 1994.

[14] S. G. Samko, A. A. Kilbas, and O. I. Marichev, Fractional Integrals and Derivatives, Gordon and Breach, Amsterdam, The Netherlands, 1993.

[15] I. Podlubny, Fractional Differential Equations, Academic Press, San Diego, Calif, USA, 1999.

[16] K. B. Oldham and J. Spanier, The Fractional Calculus, Academic Press, New York, NY, USA, 1974.

[17] D. Băleanu, K. Diethelm, E. Scalas, and J. J. Trujillo, Fractional Calculus: Models and Numerical Methods, Series on Complexity, Nonlinearity and Chaos, World Scientific, Boston, Mass, USA, 2012.

[18] K. S. Miller and B. Ross, An Introduction to the Fractional Calculus and Fractional Differential Equations, John Wiley \& Sons, New York, NY, USA, 1993.

[19] A. A. Kilbas, H. M. Srivastava, and J. J. Trujillo, Theory and Applications of Fractional Differential Equations, Elsevier, Amsterdam, The Netherlands, 2006.

[20] F. Mainardi, Fractional Calculus and Waves in Linear Viscoelasticity, Imperial College Press, London, UK, 2010.

[21] M. Y. Xu and W. C. Tan, "Theoretical analysis of the velocity field, stress field and vortex sheet of generalized second order fluid with fractional anomalous diffusion," Science in China A, vol. 44, no. 11, pp. 1387-1399, 2001.

[22] C. P. Li, W. H. Deng, and D. Xu, "Chaos synchronization of the Chua system with a fractional order," Physica A, vol. 360, no. 2, pp. 171-185, 2006.

[23] J.-S. Duan, "Time- and space-fractional partial differential equations," Journal of Mathematical Physics, vol. 46, no. 1, Article ID 013504, pp. 13504-13511, 2005.
[24] J.-S. Duan, "The periodic solution of fractional oscillation equation with periodic input," Advances in Mathematical Physics, vol. 2013, Article ID 869484, 6 pages, 2013.

[25] J. S. Duan, R. Rach, D. Baleanu, and A. M. Wazwaz, "A review of the Adomian decomposition method and its applications to fractional differential equations," Communications in Fractional Calculus, vol. 3, no. 2, pp. 73-99, 2012.

[26] F. Liu, P. Zhuang, V. Anh, I. Turner, and K. Burrage, "Stability and convergence of the difference methods for the space-time fractional advection-diffusion equation," Applied Mathematics and Computation, vol. 191, no. 1, pp. 12-20, 2007.

[27] Z. H. Wang and X. Wang, "General solution of the BagleyTorvik equation with fractional-order derivative," Communications in Nonlinear Science and Numerical Simulation, vol. 15, no. 5, pp. 1279-1285, 2010.

[28] A. M. Yang, C. Cattani, H. Jafari, and X. J. Yang, "Analytical solutions of the onedimensional heat equations arising in fractal transient conduction with local fractional derivative," Abstract and Applied Analysis, vol. 2013, Article ID 462535, 5 pages, 2013.

[29] G. W. Bluman and S. C. Anco, Symmetry and Integration Methods for Differential Equations, Springer, New York, NY, USA, 2002.

[30] R. Gorenflo, Y. Luchko, and F. Mainardi, "Wright functions as scale-invariant solutions of the diffusion-wave equation," Journal of Computational and Applied Mathematics, vol. 118, no. 1-2, pp. 175-191, 2000.

[31] W. Wyss, "The fractional diffusion equation," Journal of Mathematical Physics, vol. 27, no. 11, pp. 2782-2785, 1986.

[32] E. Buckwar and Y. Luchko, "Invariance of a partial differential equation of fractional order under the Lie group of scaling transformations," Journal of Mathematical Analysis and Applications, vol. 227, no. 1, pp. 81-97, 1998.

[33] B. Davies, Integral Transforms and Their Applications, Springer, New York, NY, USA, 3rd edition, 2002.

[34] A. M. Mathai and R. K. Saxena, The H-Function with Applications in Statistics and Other Disciplines, John Wiley \& Sons, New Delhi, India, 1978.

[35] H. M. Srivastava, K. C. Gupta, and S. P. Goyal, The H-Functions of One and Two Variables with Applications, South Asian, New Delhi, India, 1982. 


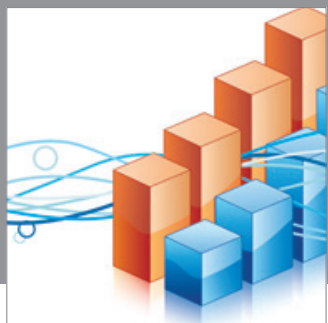

Advances in

Operations Research

mansans

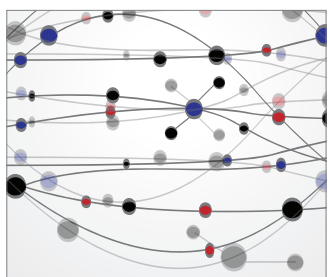

The Scientific World Journal
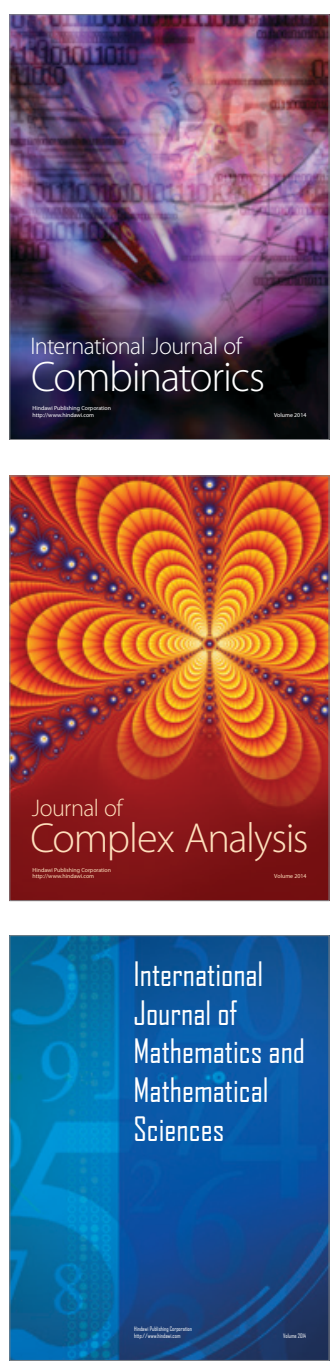
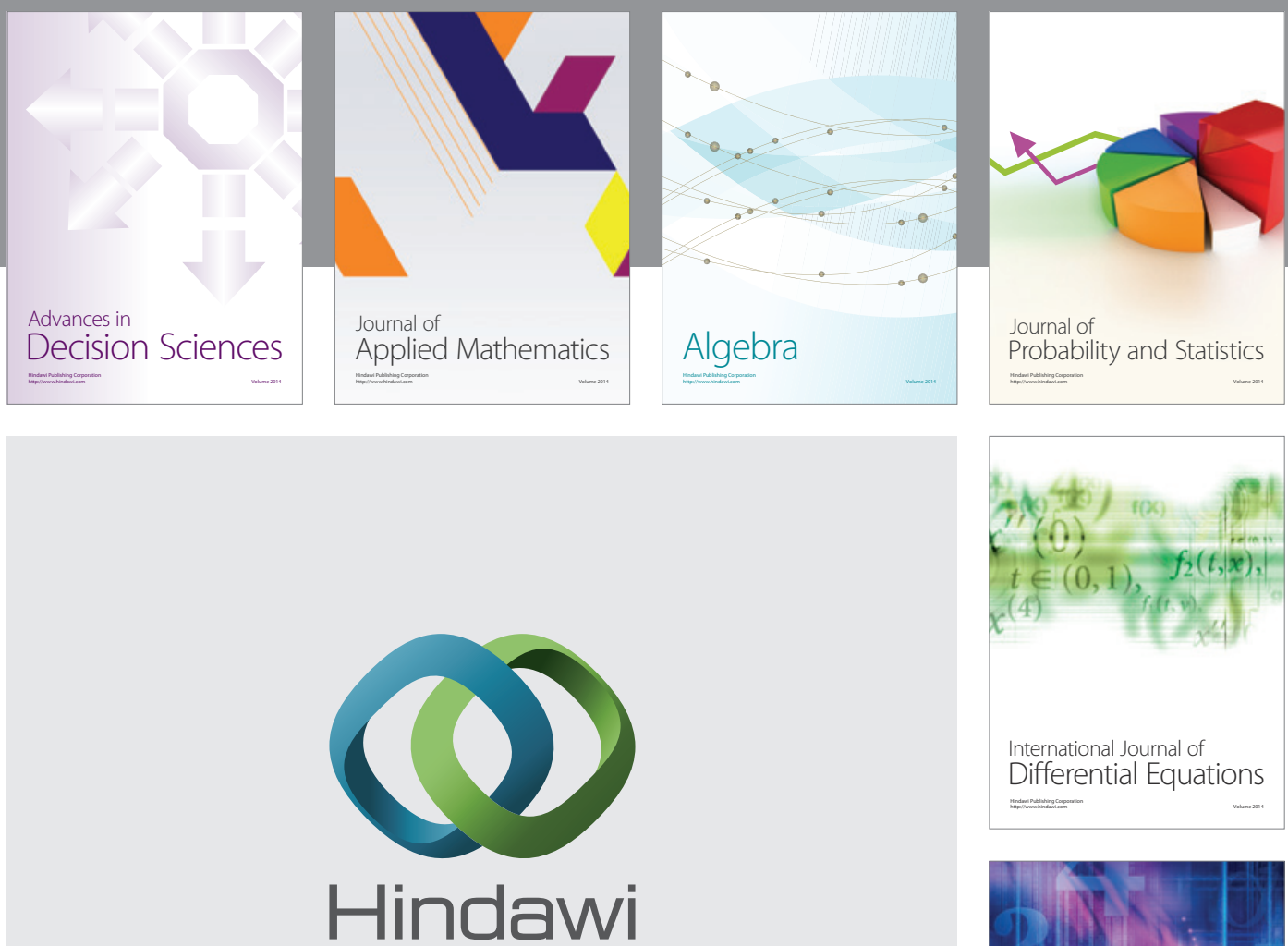

Submit your manuscripts at http://www.hindawi.com
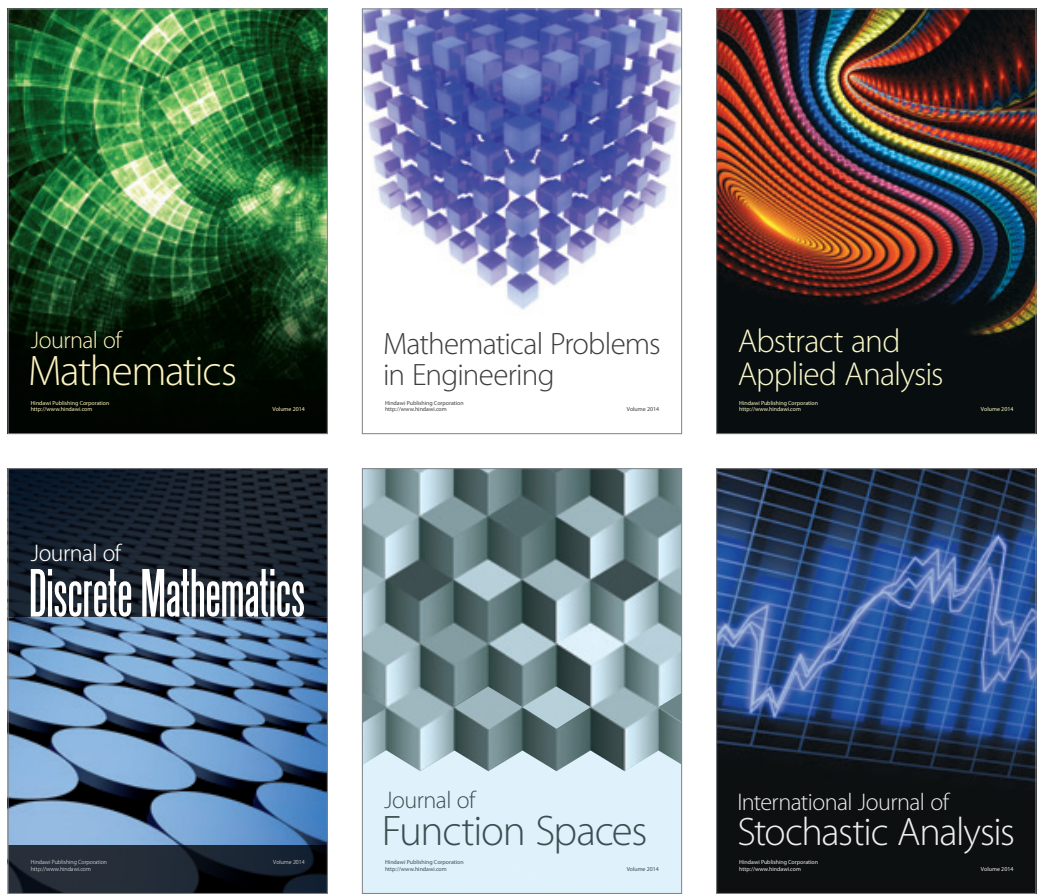

Journal of

Function Spaces

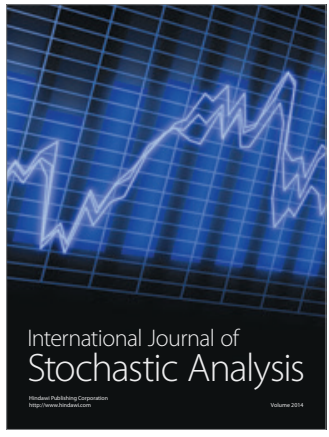

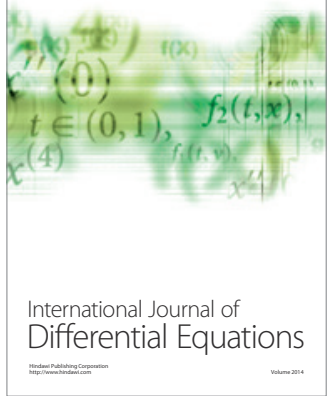
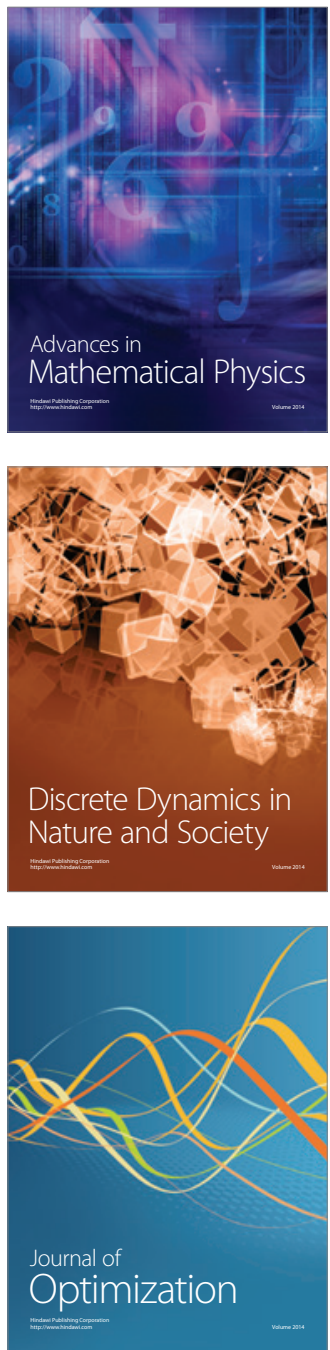